\section{Clinical insights for early detection of acute transverse myelitis in the emergency department}

\author{
Yo Huh, Eun-Jung Park, Ju-Won Jung, Sungho Oh, Sang-Cheon Choi \\ Department of Emergency Medicine, Ajou University School of Medicine, Suwon, Korea
}

Objective Acute transverse myelitis (ATM) is characterized by motor weakness, sensory changes, and autonomic dysfunction. However, diagnosis of ATM is based on early-stage clinical features only (and clarification of the cause of disease), which are difficult for emergency department (ED) physicians owing to low incidence rates. We performed retrospective analysis of ATM in order to provide clinical insights for early detection.

Methods Medical records of patients, who were finally diagnosed with ATM from January 2005 to February 2013, were investigated. Data, including demographics, clinical findings, and radiographic findings, were reviewed.

Results Forty-six patients were included in the present study, with a mean age of 43.4 years. Sensory changes were identified in 45 patients (97.8\%), motor weakness in 33 patients (71.7\%), and autonomic dysfunction in 35 patients (76.1\%). Thirty patients (65.2\%) showed high signal intensity in T2-weighted magnetic resonance imaging (MRI), with lesions most frequently found in the thoracic level of the spinal cord (56.7\%). There were discrepancies between sensory changes and levels of MRI lesions. Thirty-five patients (76.1\%) were diagnosed with idiopathic ATM. Initial diagnostic impressions in the ED were herniated intervertebral disc (38.7\%), stroke (19.4\%), Guillain-Barré syndrome (12.9\%), cauda equina syndrome (9.7\%), ATM (9.7\%), and others (9.7\%).

Conclusion When a patient presents with motor weakness, sensory changes, or autonomic dysfunction, ATM should be initially considered as a differential diagnosis, unless the ED physician's impression after initial evaluation is clear.

Keywords Myelitis, transverse; Emergency service, hospital; Diagnosis

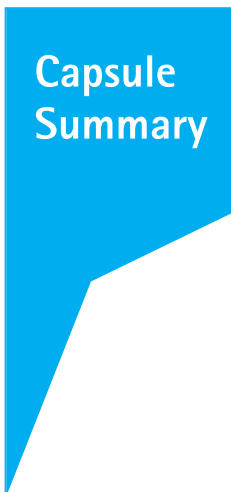

What is already known

ATM is characterized by motor, sensory, and autonomic spinal cord dysfunctions. It is difficult for clinicians to diagnose ATM based on clinical features at presentation. Clinical diagnostics have shown low sensitivity and low specificity.

What is new in the current study

Although typical symptoms and sign of ATM were not shown, if any of the bilateral/unilateral sensory abnormalities, bilateral/unilateral motor dysfunctions or autonomic dysfunctions exist in patients, ED clinicians should recall that ATM is one of the differential diagnosis
elSSN: 2383-4625

Received: 15 August 2014 Revised: 10 December 2015 Accepted: 11 December 2015

Correspondence to: Sang-Cheon Choi Department of Emergency Medicine, Ajou University School of Medicine, 206 World cup-ro, Yeongtong-gu,

Suwon 443-721, Korea

E-mail: avenue59@ajou.ac.kr

The first two authors contributed equally to the present study.

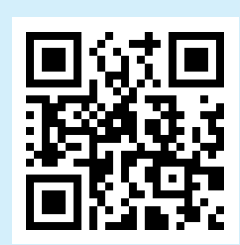

How to cite this article:

Huh Y, Park EJ, Jung JW, Oh S, Choi SC. Clinical insights for early detection of acute transverse myelitis in the emergency department. Clin Exp Emerg Med 2015;2(1):44-50.

This is an Open Access article distributed under the terms of the Creative Commons Attribution Non-Commercial License (http:// creativecommons.org/licenses/by-nc/3.0/). 


\section{INTRODUCTION}

Acute transverse myelitis (ATM) is a myelopathy that is characterized by acute or sub-acute motor, sensory, and autonomic spinal cord dysfunctions. ${ }^{1-3}$ The pathophysiology of ATM remains unclear, but may be caused by a block of ascending and descending spinal tracts at specific levels of the spinal cord. ${ }^{1}$ Symptoms and signs of motor, sensory, and autonomic nervous dysfunction may occur simultaneously, but their occurrence depends on the spinal cord levels involved. ${ }^{3-5}$ ATM develops typically over the course of hours to days, with a longer course possible in some cases. ${ }^{1,3,6}$ Bilateral symptoms and signs below the level of the spinal cord lesion, characterized by clear sensory demarcation, can occur. ${ }^{1,3,6}$ In ATM, clinical symptoms and signs repeatedly and additionally occur for different reasons. One-third of patients with ATM recover completely, another one-third show residual neurologic deficits that are clinically moderate, and the remaining onethird show residual neurologic deficits that are clinically severe. Prognosis is poor when ATM shows a rapid course of progression or spinal shock. ${ }^{2,7}$ Therefore, early detection and treatment decisions determine the prognosis of ATM. ${ }^{6}$

Previous studies report that incidence rates of ATM vary in range from 1.34 to 4.6 per million. ${ }^{1,3,6}$ Because of the low incidence rate, emergency department (ED) physicians lack familiarity with ATM. It can be difficult for ED physicians to consider the diagnosis of ATM for patients with atypical clinical features at ED admission. Therefore, we think it is important for ED physicians to be well aware of the disease entity in advance. We performed retrospective analysis of cases finally diagnosed with ATM from January 2005 to February 2013 to provide ED physicians with clinical insights for early detection.

\section{METHODS}

\section{Study design and setting}

Patients who were admitted to the ED at Ajou University Hospital and were finally diagnosed with ATM from January 2005 to February 2013 were included in the present study. This study was approved by our institutional review board, which deemed it exempt from the informed consent requirement. We used the following diagnostic criteria for ATM: 1) bilateral sensorimotor and autonomic spinal cord dysfunctions that are not necessarily symmetric; 2) clearly defined sensory level; 3) progression to nadir of clinical deficits between 4 hours and 21 days after the onset of symptoms; 4) demonstration of spinal cord inflammation (e.g., cerebrospinal fluid pleocytosis, elevated IgG index, or magnetic resonance imaging [MRI] revealing a gadolinium-enhancing cord le- sion); and 5) exclusion of compressive, postradiation, neoplastic, and vascular causes. ${ }^{1,7}$ We investigated retrospectively the medical records of patients with regard to sex, age, medical history, course of disease, symptoms and signs, results of physical examinations (including neurologic examinations), initial impression in the ED, time from ED admission to clinical department referral, laboratory results (including spinal tapping), neuroimaging results, and clinical course.

\section{Data collection}

Standardized extraction of demographic, clinical, laboratory, and radiological data from medical records was performed by 2 trained ED physicians. Any discrepancy between the 2 sets of data extracted was resolved by a third physician.

Sensory abnormality was defined as "positive" when a sensory change was observed and "negative" when a subjective sensory change was observed, but the neurologic examination was normal. The modified Medical Research Council scale was used to evaluate motor functions. ${ }^{8}$ Autonomic dysfunction was defined as "positive" when the patient had medical record documentation of urinary urgency, urinary incontinence, fecal incontinence, tenesmus, or sexual dysfunction.

We investigated test results of blood that had been sampled at ED admission, such as anti-nuclear antibody, antibodies for human immunodeficiency virus and hepatitis virus, venereal disease research laboratory test, and polymerase chain reaction for tuberculosis. Lumbar puncture records were reviewed for opening pressure, cytology, protein level, and IgG index. Brain computed tomography and MRI images were reviewed, and the results were confirmed by radiologists. Spinal MRI records were reviewed for the site and length of the involved lesion, multiplicity of lesion, and gadolinium enhancement of lesion. The following were also investigated: steroid administration, plasmapheresis therapy, potential causes of ATM, time from ER admission to clinical department referral, and sequelae at hospital discharge.

\section{Statistical analysis}

All medical record data were transformed and coded using SPSS ver. 18.0 (SPSS Inc., Chicago, IL, USA). For descriptive analysis, all data were expressed as the mean \pm standard deviation or the median (interquartile range, IQR) as appropriate.

\section{RESULTS}

\section{Clinical characteristics}

Forty-six subjects were included in the present study (Table 1). Mean age was $43.4 \pm 15.3$, with a sizeable portion of subjects 
Table 1. Demographics and clinical features

\begin{tabular}{|c|c|}
\hline Characteristic & No. $(\%)$ \\
\hline Age (yr), mean \pm SD & $43.4 \pm 15.29$ \\
\hline$\leq 10$ & $3(6.5)$ \\
\hline $11-20$ & $0(0.0)$ \\
\hline $21-30$ & $5(10.9)$ \\
\hline $31-40$ & $9(19.6)$ \\
\hline $41-50$ & $12(26.1)$ \\
\hline $51-60$ & $10(21.7)$ \\
\hline $61-70$ & $6(13.0)$ \\
\hline$\geq 71$ & $1(2.2)$ \\
\hline \multicolumn{2}{|l|}{ Sex } \\
\hline Male & $23(50.0)$ \\
\hline Female & $23(50.0)$ \\
\hline \multicolumn{2}{|l|}{ Past medical history } \\
\hline Transverse myelitis & $2(4.5)$ \\
\hline Connective tissue disease & $1(2.3)$ \\
\hline Recent episode of upper respiratory infection & $7(15.9)$ \\
\hline Recent episode of vaccination & $0(0)$ \\
\hline Time (day) before reaching a medical facility, median (IQR) & $3(9)$ \\
\hline Time (day) to maximal deficit, median (IOR) & $1(4.5)$ \\
\hline Presence of progressive symptom & $37(80.4)$ \\
\hline \multicolumn{2}{|l|}{ Referral impression from emergency department } \\
\hline Herniated intervertebral disc & $12(38.7)$ \\
\hline Stroke & $6(19.4)$ \\
\hline Guillain-Barré syndrome & $4(12.9)$ \\
\hline Cauda equina syndrome & $3(9.7)$ \\
\hline Acute transverse myelitis & $3(9.7)$ \\
\hline Others & $3(9.7)$ \\
\hline \multicolumn{2}{|l|}{ Symptoms } \\
\hline Fever & $1(2.2)$ \\
\hline Back pain & $17(37.0)$ \\
\hline Sensory deficit & $45(97.8)$ \\
\hline Motor deficit & $33(71.7)$ \\
\hline Urinary dysfunction & $25(54.3)$ \\
\hline Bowel dysfunction & $9(19.6)$ \\
\hline Sexual dysfunction & $1(2.2)$ \\
\hline \multicolumn{2}{|l|}{ Treatment } \\
\hline Steroid pulse therapy & 45 (97.8) \\
\hline Immunoglobulin & $4(8.7)$ \\
\hline Plasmapheresis & $5(10.9)$ \\
\hline Others & $3(6.5)$ \\
\hline \multicolumn{2}{|l|}{ Final diagnosis } \\
\hline Idiopathic & $35(76.1)$ \\
\hline Viral infection & $5(10.9)$ \\
\hline Mixed connective tissue disease & $1(2.2)$ \\
\hline Systemic sclerosis & $3(6.5)$ \\
\hline Others & $2(4.3)$ \\
\hline Presence of sequelae at discharge & $44(95.7)$ \\
\hline Sensory sequelae & $35(76.1)$ \\
\hline Motor sequelae & $26(56.5)$ \\
\hline Autonomic system sequelae & $21(45.7)$ \\
\hline
\end{tabular}

$I Q R$, interquartile range.

(47.8\%) being in their forties and fifties. Seven subjects (15.9\%) had upper respiratory infections, but none had been vaccinated for any product of vaccination recently according to medical history. Disease progression was identified in 37 subjects (80.4\%),
Table 2. Characteristics of motor and sensory deficits

\begin{tabular}{lc}
\hline Characteristic & No. (\%) \\
\hline Sensory deficit & \\
None & $1(2.2)$ \\
Unilateral & $9(19.6)$ \\
Bilateral & $36(78.3)$ \\
Any involvement of cervical spinal cord & $8(17.8)$ \\
Any involvement of thoracic spinal cord & $34(75.6)$ \\
Any involvement of lumbar spinal cord & $37(80.4)$ \\
Any involvement of sacral spinal cord & $29(64.4)$ \\
Cervical+thoracic & $2(4.4)$ \\
Thoracic+lumbar & $2(4.4)$ \\
Thoracic+sacral & $1(2.2)$ \\
Lumbar+sacral & $4(8.9)$ \\
Cervical+thoracic+lumbar & $5(11.1)$ \\
Thoracic+lumbar+sacral & $24(53.3)$ \\
Motor deficit & \\
None & $13(28.3)$ \\
Unilateral & $8(17.4)$ \\
Bilateral & $25(54.3)$ \\
Any involvement of shoulder joint & $4(12.1)$ \\
Any involvement of elbow joint & $7(21.2)$ \\
Any involvement of wrist joint & $7(21.2)$ \\
Any involvement of hip joint & $31(93.9)$ \\
Any involvement of knee joint & $31(93.9)$ \\
Any involvement of ankle joint & $30(90.9)$ \\
Hip+knee+ankle & $20(60.6)$ \\
Elbow+wrist+knee+ankle & $1(3.0)$ \\
Elbow+hip+knee+ankle & $3(9.1)$ \\
Wrist+hip+knee+ankle & $2(6.1)$ \\
Shoulder+elbow+wrist+hip+knee+ankle & $4(12.1)$ \\
\hline &
\end{tabular}

and the mean time from development to full-blown symptoms was 1.0 day $(4.5, I Q R)$. Sensory abnormality was observed in 45 subjects (97.8\%), motor abnormality in 33 subjects $(71.7 \%)$, and autonomic dysfunction in 25 subjects (54.3\%). Idiopathic ATM was observed most commonly, in 35 of the subjects (76.1\%). Forty-five subjects (97.8\%) received intravenous steroid administration, and 5 (10.9\%) received plasmapheresis. Residual neurological deficits at hospital discharge were identified in 44 subjects $(95.7 \%)$.

\section{Characteristics of sensory and motor abnormalities}

Among the 45 subjects showing sensory abnormality, 36 subjects (80.0\%) showed bilateral abnormality (Table 2). Sensory abnormality at the lumbar spinal cord level was identified in 37 subjects (80.4\%), at the thoracic spinal cord level in 34 subjects (75.6\%), and at the sacral spinal cord level in 29 subjects (64.4\%). Second lumbar and fifth lumbar levels were the most common, affecting 35 subjects (77.8\%) (Fig. 1). Eleven subjects (24.4\%) had sensory abnormalities confined to just one level of the cervical, thoracic, lumbar, or sacral spinal cord, with the lumbar spinal cord level the most common (5 of the 11 subjects, 11.1\%). Thoracic, lumbar, and sacral spinal cord were simultaneously involved in 24 sub- 

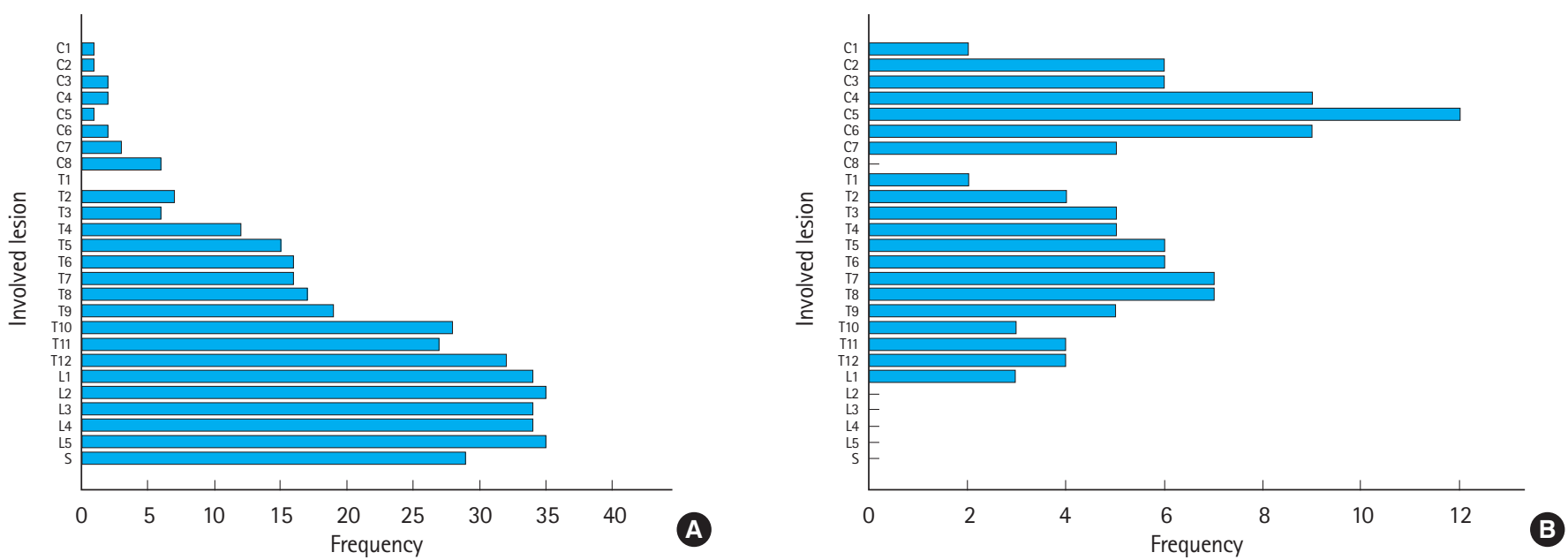

Fig. 1. Comparison of sensory deficits and magnetic resonance imaging (MRI) lesions. Most sensory deficits (A) were observed in levels of thoracic and lumbar spinal cord. However, most MRI lesions (B) were observed in levels of cervical and thoracic spinal cord. There were discrepancies between sensory deficits and MRI lesions.

jects (53.3\%) (Table 2).

Among the 33 subjects showing motor abnormality, 25 subjects (75.8\%) showed bilateral abnormality. Motor abnormalities were mainly identified in lower extremities. Thirty-one subjects had abnormalities in the hip joint and knee joint (93.9\% for each joint), and 30 subjects (90.9\%) had abnormalities in the ankle joint.

Thirty-two subjects (69.6\%) showed both sensory and motor abnormalities; 24 subjects (52.2\%) showed both sensory abnormality and autonomic dysfunction; 22 subjects (47.8\%) showed both motor abnormality and autonomic dysfunction; and 21 subjects (45.7\%) showed abnormalities in all three (sensory, motor, and autonomic) systems.

\section{Initial diagnostic impression and time from emergency department admission to referral}

For this analysis, fifteen of the 46 subjects were excluded either because subjects were consulted to neurologist or neurosurgeon for an impression of ATM, or because neurology or neurosurgery directly managed patient following the initial ED admission. For the 31 subjects, the initial diagnostic impressions in the ED were: 1) herniated intervertebral disc (12 subjects, 38.7\%); 2) stroke (6 subjects, 19.4\%); 3) Guillain-Barré syndrome (4 subjects, 12.9\%); 4) cauda equina syndrome (3 subjects, 9.7\%); 5) ATM (3 subjects, 9.7\%); and 6) others (3 subjects, 9.7\%). Median time from ED admission to referral was 68.5 minutes (IQR, 30.0 to 165.5$)$.

\section{Characteristics of magnetic resonance imaging and spinal tapping studies}

With regard to MRI findings, typical findings of ATM were identi-
Table 3. Characteristics of magnetic resonance imaging findings and cerebrospinal fluid analysis

\begin{tabular}{lc}
\hline Characteristic & No. (\%) \\
\hline Spinal magnetic resonance imaging & \\
Normal & $7(15.2)$ \\
Compatible with ATM & $30(65.2)$ \\
Other abnormal findings & $9(19.6)$ \\
Spinal magnetic resonance imaging compatible with ATM & \\
Abnormality in T1 & $1(3.3)$ \\
Abnormality in T2 & $30(100)$ \\
T1 gadolinium enhancement & $25(83.3)$ \\
Abnormality in T1 gadolinium enhancement & $13(43.3)$ \\
Any involvement of cervical spinal cord & $16(53.3)$ \\
Any involvement of thoracic spinal cord & $17(56.7)$ \\
Any involvement of lumbar spinal cord & $3(10.0)$ \\
Any involvement of sacral spinal cord & $0(0.0)$ \\
CSF analysis & \\
Opening pressure (cmH ${ }_{2}$ O), median (IQR) & $14.0(6.63)$ \\
CSF pleocytosis & $13(28.3)$ \\
Protein (mg/dL), median (IOR) & $42.5(43.38)$ \\
Glucose (mg/dL), median (IOR) & $59.5(61.88)$ \\
IgG index (mg/dL), median (IOR) & $4.0(4.88)$ \\
\hline
\end{tabular}

ATM, acute transverse myelitis; IQR, interquartile range; CSF, cerebrospinal fluid.

fied in 30 subjects (65.2\%), and non-specific myelopathy and signal changes were identified in 9 subjects (19.6\%) (Table 3). Among the 30 subjects with typical ATM findings, all showed high signal intensity in T2-weighted images, and gadolinium enhancement of the lesion was identified in 13 subjects (43.3\%). Seventeen subjects (56.7\%) showed abnormalities in the thoracic level of the spinal cord, and 16 subjects (53.3\%) showed abnormalities in the cervical level of the spinal cord. Spinal cord lesions were found at the 5th cervical cord level in 12 subjects (40\%), and at the 4th and 6th cervical cord levels in 9 subjects each (30\% for each). Twenty-six subjects (86.7\%) had lesions limited to just one spinal 
cord level, while 4 subjects had lesions involving multiple sites of the spinal cord (13.3\%). Among all included subjects, 34 subjects (73.9\%) had brain MRIs, 26 of whom (76.4\%) had normal findings. For the 8 subjects with abnormal findings, 3 subjects had non-specific signal changes, 4 subjects had diffuse cortical atrophy, and 1 subject had suspicious findings of multiple sclerosis.

The lumbar puncture results identified leukocytosis in 13 subjects (28.3\%), pleocytosis and increased levels of protein in 24 subjects (52.2\%), and increased lgG index levels in 11 subjects (23.9\%) (Table 3).

\section{DISCUSSION}

Because rapid progression and spinal shock in the course of ATM denote poor prognosis, rapid identification and early treatment are paramount for effective ATM treatment. ${ }^{6,7}$ However, it is difficult for clinicians to diagnose ATM based on clinical features at presentation, and to differentiate the etiology clearly during early-stage ATM. ${ }^{2,3,5}$ In addition, the diagnostic criteria for idiopathic ATM established by the Transverse Myelitis Consortium Working Group (TMCWG) has been reported to be of limited use. Finally, clinical diagnostics, including laboratory and radiologic tests for differentiation of etiology, have shown low sensitivity and low specificity.

Although previous studies regarding ATM have reported the epidemiology, cause, diagnosis, treatment, and prognosis of ATM, more information remains to be elucidated for a prompt referral from the ED to a specialist. The incidence rate of ATM is rare, and the main complaints related to ATM are similar to the initial symptoms and signs of a variety of diseases frequently seen in the ED. Thus, there are often delays in deciding to refer patients who present to the ED with a few mere symptoms and signs of ATM. Common ATM symptoms and signs include acute or sub-acute sensory changes and motor abnormality, back pain, spinal cord dysfunction, and autonomic dysfunction, such as urinary and fecal incontinence. ${ }^{1,9,10}$ Because these symptoms and signs are similar to the main complaints of cauda equina syndrome, conus medullaris syndrome, herniated intervertebral disc, stroke, and GuillainBarré syndrome, early consideration of ATM would be rare for ED physicians without prior knowledge of ATM. As shown in Table 1, ATM was the initial diagnostic impression for just 3 of 31 cases $(9.7 \%)$, which means there was some delay in diagnosing the other ATM cases. Early diagnosis is closely related with a good prognosis, so enhancing prior knowledge and ability to recognize ATM among ED physicians is needed. Although typical symptoms and signs of ATM were not shown, if any of the bilateral/unilateral sensory abnormalities, bilateral/unilateral motor dysfunctions, or autonomic dysfunctions exist in patients, ED clinicians should recall that ATM is one of the differential diagnoses. Concise information from the clinical history of the patient; results of laboratory tests, including an analysis of cerebrospinal fluid; and brain and spinal MRls may be needed for differential diagnosis. Even if MRI findings are non-specific or normal, "possible ATM" at an early stage of progress should still be considered. The exact incidence and prevalence of ATM has not been established to date. According to previous studies, the mean age of ATM ranges from 41 to 45 years, with a bi-modal peak distribution around 10 to 19 years and 30 to 39 years. ${ }^{1,6,9-11}$ However, the mean age in the present study was 43.4 years, and the commonly affected ages were between 41 and 60 years old.

The cause of ATM has been attributed largely to demyelinating diseases and other inflammatory disease groups. ${ }^{2}$ Among demyelinating diseases, idiopathic ATM is the most common, with incidence ranging from $9 \%$ to $60 \%{ }^{2}$ Previous studies reported that $46 \%$ of ATM patients had previous viral infections and related symptoms, including respiratory and gastrointestinal systems, ${ }_{1}^{2,3,9-11}$ however, no specific virus is a known cause of ATM. ${ }^{12}$ In the present study, idiopathic ATM was the most common type (76.1\%); the percentage was higher than in other studies, and subjects with previous related viral or direct infections were 10.9\% of the total. Therefore, idiopathic ATM should be one of the top differential diagnoses when patients complain of sensory changes, motor weakness, and/or autonomic dysfunction at ED admission (even with no previous viral infection).

In previous studies, initial symptoms of ATM were pain or sensory changes/deficits, which developed in the back, abdomen, or extremities. $^{3-5,13}$ Pain could develop at the midline of the trunk; however, pain may develop along the dermatome of the spinal cord. Sensory changes occurred in $80 \%$ to $94 \%$ of ATM. ${ }^{1,4}$ In terms of sensory change, sensory abnormalities were the most common in our study, as shown in Table 2. Lesions of sensory abnormality were most common in the lumbar and thoracic areas (80.4\% and $75.6 \%$, respectively). This was not restricted to one level of the spinal cord, but developed as variant features. We think it is important to recognize the tendencies of sensory change, which lead to a high index of suspicion of ATM in the ED.

Motor abnormalities were reported in about 50\% of total ATM in previous studies. ${ }^{1,4}$ In the present study, motor abnormality in the lower extremity was identified in $93.9 \%$ of subjects. Motor abnormalities in all joints (ankles, knees, and hips) were identified in $60.6 \%$ of subjects. Unilateral motor abnormality was identified in $19.6 \%$ of subjects who were not previously diagnosed with immunologic diseases, such as multiple sclerosis. Both sensory and motor abnormalities developed more in the lower extremities 
in previous studies. ${ }^{1,4}$ According to Kaplin et al., ${ }^{14}$ lesions at the thoracic and cervical cord level were present in 63\% and 22\%, respectively. In the present study, the proportion of subjects with motor abnormality was $71.7 \%$, which was higher than findings of previous studies, but lower than the proportion of subjects in our study with sensory changes (97.9\%). Autonomic dysfunction, including urinary incontinence, tenesmus, constipation, and sexual dysfunction affected $54.3 \%$ of subjects in the present study. This result was higher than those reported in previous studies $(2.8 \%$ to $41.1 \%)^{4,5,15}$

The purposes of radiologic evaluation for patients suspected of having ATM are to differentiate other spinal cord lesions, and to investigate the cause of ATM. ${ }^{1,2}$ The diagnostic criteria by TMCWG recommends spinal MRI evaluation with gadolinium enhancement to facilitate early identification of ATM and to determine the cause. ${ }^{7}$ However, because there were cases that showed normal findings on spinal MRI, "possible ATM" cases should be reevaluated later with spinal MRI., According to previous studies, $53 \%$ of ATM cases showed normal findings on spinal MRI, and $19 \%$ to $42.1 \%$ showed no gadolinium enhancement on spinal MRI. ${ }^{5,16}$ In the present study, 65.2\% of the subjects showed high signal intensity of T2-weighted images, and $43.3 \%$ of subjects showed gadolinium enhancement on spinal MRI. Lesions at the thoracic spinal cord level are the most common spinal lesions found by MRI. ${ }^{4,15}$ However, Kaplin et al. ${ }^{14}$ and De Seze et al. ${ }^{16}$ reported cervical spinal cord lesions in $44 \%$ and $37 \%$ of subjects, respectively, and thoracic spinal cord lesions in 60\% and 33\% of subjects, respectively. According to Kaplin et al., ${ }^{14}$ although the level of symptoms and signs was most often at the thoracic level of the spinal cord, abnormal MRI results were most common at the cervical level of the spinal cord. This difference was speculated to stem from the sensory distribution characteristics of the spinal cord. In the present study, the level of symptoms and signs was most common at the lumbar level of the spinal cord, and abnormal MRI results were most common at the thoracic level of the spinal cord (56.7\%). We think this difference between the levels of clinical symptoms and signs and the levels of abnormal $\mathrm{MRI}$ results carries a significant implication. For example, the increased tone and weakness in the lower extremities, the thoracic spine sensory level, and the lack of symptoms and signs in the upper extremities suggest a lesion in the thoracic spinal cord. Therefore, we think that if symptoms and signs of patients are suggestive of ATM, then a detailed medical history and thorough neurologic examination will be necessary to avoid false negatives in the diagnostic work-up for ATM. In addition, evaluation of an expanded spinal MRI should be considered to detect lesions above the suspected spinal level.
The present study has several limitations. First, the set of data collected was not the same for all subjects included in the study because it was a retrospective study. This may have added bias to the results of the study. Second, we do not present the reasons why there were differences between our study and previous studies in terms of age, sex, and the most common lesions, although a relatively higher number of cases were studied in ours compared with the previous studies. Third, our study lacked results from laboratory testing of diagnosis and treatment, and from long-term patient follow-up. Thus, it was difficult to critically evaluate clinical characteristics and results of diagnostic tests. Fourth, the data used in the present study were retrospectively collected at a single center, which allows for possible bias. Further studies will be needed to identify clinical features and characteristics of diagnostic tests, and to elucidate the relationship between early identification of ATM and prognosis.

Early identification and timely diagnosis of ATM is a challenge for ED physicians because ATM cases usually present with variable symptoms and signs that are similar to those of other diseases, including herniated intervertebral disc, Guillain-Barré syndrome, conus medullaris syndrome, and stroke. In addition, both MRIs, particularly those that are restricted to a specific level, and cerebrospinal fluid examination may show no abnormal findings during early-stage ATM. Therefore, ATM should be initially considered as a differential diagnosis when the patient presents with any symptoms of motor weakness, sensory changes, or autonomic dysfunction, even if they had no previous viral infections or immune-related diseases.

\section{CONFLICT OF INTEREST}

No potential conflict of interest relevant to this article was reported.

\section{REFERENCES}

1. Frohman EM, Wingerchuk DM. Clinical practice: transverse myelitis. N Engl J Med 2010;363:564-72.

2. Jacob A, Weinshenker BG. An approach to the diagnosis of acute transverse myelitis. Semin Neurol 2008;28:105-20.

3. Borchers AT, Gershwin ME. Transverse myelitis. Autoimmun Rev 2012;11:231-48.

4. Jun YH, Kim SD, Park JY, et al. Clinical characteristics of idiopathic acute transverse myelitis. Korean J Spine 2005;2:13843.

5. Han DC, Kim JH, Shin DJ, Park HM, Lee YB. Suspected idiopathic acute transverse myelitis: retrospective analysis of 27 
cases. Korean J Clin Neurophysiol 2006;8:58-62.

6. Bhat A, Naguwa S, Cheema G, Gershwin ME. The epidemiology of transverse myelitis. Autoimmun Rev 2010;9:A395-9.

7. Transverse Myelitis Consortium Working Group. Proposed diagnostic criteria and nosology of acute transverse myelitis. Neurology 2002;59:499-505.

8. Paternostro-Sluga T, Grim-Stieger M, Posch M, et al. Reliability and validity of the Medical Research Council (MRC) scale and a modified scale for testing muscle strength in patients with radial palsy. J Rehabil Med 2008;40:665-71.

9. Berman M, Feldman S, Alter M, Zilber N, Kahana E. Acute transverse myelitis: incidence and etiologic considerations. Neurology 1981;31:966-71.

10. Jeffery DR, Mandler RN, Davis LE. Transverse myelitis: retrospective analysis of 33 cases, with differentiation of cases associated with multiple sclerosis and parainfectious events. Arch Neurol 1993;50:532-5.
11. Christensen PB, Wermuth L, Hinge HH, Bomers K. Clinical course and long-term prognosis of acute transverse myelopathy. Acta Neurol Scand 1990;81:431-5.

12. Kincaid 0, Lipton HL. Viral myelitis: an update. Curr Neurol Neurosci Rep 2006;6:469-74.

13. Ropper AH, Poskanzer DC. The prognosis of acute and subacute transverse myelopathy based on early signs and symptoms. Ann Neurol 1978;4:51-9.

14. Kaplin Al, Krishnan C, Deshpande DM, Pardo CA, Kerr DA. Diagnosis and management of acute myelopathies. Neurologist 2005;11:2-18.

15. Thomas T, Branson HM, Verhey LH, et al. The demographic, clinical, and magnetic resonance imaging (MRI) features of transverse myelitis in children. J Child Neurol 2012;27:11-21.

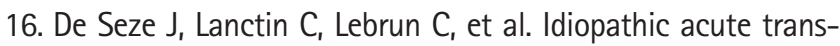
verse myelitis: application of the recent diagnostic criteria. Neurology 2005;65:1950-3. 